\title{
An Integrated Approach of Multi-objective Optimization Model for Evaluating New Supporting Program in Irish Hospitals
}

\author{
Wael Rashwan \\ Technological University Dublin, wael.rashwan@tudublin.ie \\ Heba Habib \\ Technological University Dublin \\ Amr Arisha \\ Technological University Dublin, amr.arisha@tudublin.ie
}

See next page for additional authors

Follow this and additional works at: https://arrow.tudublin.ie/buschmarcon

Part of the Business Administration, Management, and Operations Commons

\section{Recommended Citation}

Rashwan, W. et al. (2016). An Integrated Approach of Multi-objective Optimization Model for Evaluating New Supporting Program in Irish Hospitals, Proceedings of the 2016 Winter Simulation Conference, Washington DC, 11th -14th December.

This Conference Paper is brought to you for free and open access by the School of Marketing at ARROW@TU Dublin. It has been accepted for inclusion in Conference papers by an authorized administrator of ARROW@TU Dublin. For more information, please contact arrow.admin@tudublin.ie, aisling.coyne@tudublin.ie, gerard.connolly@tudublin.ie.

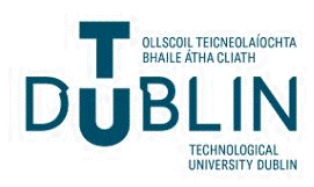


Authors

Wael Rashwan, Heba Habib, Amr Arisha, Garry Courtney, and Sean Kennelly

This conference paper is available at ARROW@TU Dublin: https://arrow.tudublin.ie/buschmarcon/156 


\title{
AN INTEGRATED APPROACH OF MULTI-OBJECTIVE OPTIMIZATION MODEL FOR EVALUATING NEW SUPPORTING PROGRAM IN IRISH HOSPITALS
}

\author{
Wael Rashwan \\ Heba Habib \\ Amr Arisha \\ Garry Courtney \\ 3S Group, College of Business, \\ Dublin Institute of Technology, (DIT) \\ Dublin, IRELAND \\ National Acute Medicine Program (AMP) \\ Health Services Executives (HSE) \\ Dublin, IRELAND \\ Sean Kennelly \\ Acute Medical Unit (AMU) \\ Tallaght Hospital \\ Dublin, IRELAND
}

\begin{abstract}
Hospitals are witnessing an inexorable growth in emergency admissions, which results in overcrowding and a poorer patient experience. The Acute Medicine Program (AMP) is one of the programs developed by the Irish health authority aimed at improving patient experience. To review the AMP intervention, this study applies a model that integrates three analytical approaches: simulation, multivariate factor analysis and multi-objective optimization. The simulation identified 14 different factors affecting five responses that were used to develop a Design of Experiments (DoE). Multivariate factor analysis used the DoE to determine the factors creating 'bottlenecks', such as downstream resources. The multi-objective optimization model, based on the Simulated Annealing approach, is applied to support management decisions on optimizing key parameters affecting the treatment journey of patients. A Pareto set of solutions found that an increase in downstream capacity and unit staff can lead to a $25 \%$ decrease at least in the patient's experience time.
\end{abstract}

\section{INTRODUCTION}

Over the past decade, healthcare systems have faced many challenges in their attempts to improve system performance. Rapidly increasing costs along with the growing demand on Emergency Departments (EDs) have put healthcare decision-makers under constant pressure to manage and control their system in a more efficient and effective way. Overcrowding in EDs, which has reached crisis proportions internationally, negatively affects patient safety, quality of care, and patient experience (Graff 1999). In 2006, ED overcrowding was declared a "national emergency" in Ireland. In November 2015, emergency presentations had increased by 15,170 compared to the same period in 2014 according to the Health Service Executive's (HSE 2015). Additionally, prolonged waiting times were reported, with more than 100 patients waiting on trolleys for hospital admission every day, and $23 \%$ of patients waiting for more than 24 hours (HSE 2015). Although Ireland is not the only country that experiences prolonged waiting and overcrowding (Forero et 
al. 2010; Bond et al. 2007; Schafermeyer and Asplin 2003), it is important not to underestimate the potential consequences this situation can have on patients' experience and safety, as well as on staff.

The National Acute Medicine Program (AMP) is a clinician-led initiative incorporating the Irish Health Service Executive's (HSE) Clinical Strategy and Programs Directorate, and the Royal College of Physicians of Ireland (RCPI) among others (HSE 2010). It has developed a framework to mitigate the pressure on EDs and to minimize the length of stay (LOS) to reduce overcrowding, by introducing specialized units working in parallel with the EDs. The framework's aim is to provide medical patients presenting to the ED with a fast track to decisions regarding their treatment journey in the hospital. Patients presented in these units get to see a senior medical doctor, who is able to make treatment decisions within almost one hour of admission.

However, hospitals managers and executives requested a formal assessment on how these units can cope with the unpredicted increase in workload and demand. In response, a project was carried out within the HSE to provide the managers with a tool to enable assessment of a dedicated unit within a hospital, and to propose a model to assist with resource planning. A Discrete-Event Simulation (DES) model is developed and validated to model the underlying relationships between such a unit and the ED. This model is then integrated with statistical analysis and optimization components to identify the significant factors that affect patient-related performance measures and to then optimize the key parameters to improve the system.

Simulation models have been successfully used to evaluate healthcare programs, policies and initiatives. In the UK, DES is used to assess one of the National Health Service's initiatives, 'See and Treat', a fasttrack method based on the principle that one clinician can see, treat and discharge a patient after initial assessment (Davies 2007). The dynamic capabilities of simulation enable more accurate interpretation of the use of hospital resources (Thorwarth, Rashwan, and Arisha 2015), supporting hospital managers in their decisions on bed usage and patient flow (Harper 2002). However, research has found that simulation modelling can be time-consuming since multiple simulations have to be carried out to achieve statistically valid results (Brailsford 2006). These obstacles, however, can be alleviated by using integrated simulation surrogate models (metamodels). The integration can provide users with the required simplicity in using the tool and also the details of the system modelling (Poropudas and Virtanen 2011). Simulation surrogate modelling has several practical benefits: one particular advantage is its sensitivity analysis, which can be used to validate a simulation model when no historical data exists, and also in measuring the impact of uncertain variables on the system being simulated (Kleijnen 2005). Another objective of developing surrogate models is to enable optimization: to find the system configuration that identifies the optimal setting of the objective function (Barton and Meckesheimer 2006; Dellino, Kleijnen, and Meloni 2009). Metamodel techniques use analytical approaches to approximate objective functions so that the metamodel can replace part or all of a simulation model with a mathematical function that reflects the input-output behaviour of that part. Integrating metamodels in this way can shorten model computation times, and consequently, simplify the whole optimization process (Reis dos Santos and Reis dos Santos 2009).

\section{ACUTE MEDICAL UNITS (AMU)}

Acute Medical Units (AMUs) were set up in many acute healthcare trusts in the UK as the result of investment in acute medicine and to alleviate pressure from the EDs. These units act as a focal point for the rapid assessment of acutely ill medical patients referred to the hospital (Oddoye et al. 2009). In Ireland, AMUs have been established in all the main hospitals to facilitate the immediate medical assessment, diagnosis and treatment of patients who suffer from a wide range of medical conditions. AMUs are not designed to play a different role from EDs; they deal with medical patients who are triaged as urgent or very urgent, while ED deals with medical and non-medical patients with different acuity levels. Every AMU should have access to a senior clinical decision-maker at all times. The benefits of AMUs arise from the efficient streaming of medical patients to a location where they can be seen without delay by a senior medical doctor (i.e. a consultant, specialist registrar or registrar). If admission is required, this will occur within a defined period, and the patient will be admitted to the most appropriate clinical area in the hospital. Patients access to these units based on their acuity level and other criteria that should be set by each hospital. 
AMUs are usually divided into two sub-units: Acute Medical Assessment Unit (AMAU) and Short Stay Unit (SSU). While the AMAU acts as the first gateway for acute medical patients referred from the ED, the SSU is for patients who need to be admitted to the hospital, but their estimated length of stay is below a certain threshold. Patients can also be admitted directly to hospital clinical wards from AMU (Figure 1).

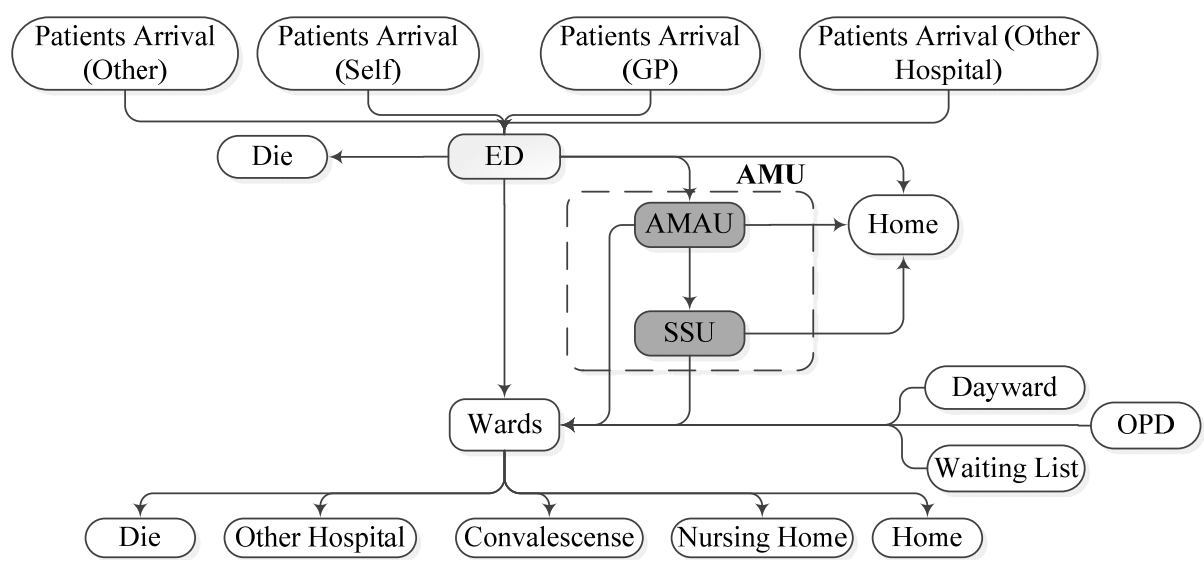

Figure 1: Generic patient's pathways through hospital with the AMU.

\section{UNDERSTANDING THE DYNAMICS}

Healthcare executives sought to gain insights into the dynamics of the ED and AMU care system applied in Tallaght Hospital, one of Ireland's largest hospitals. Recently the hospital adopted the AMP's recommendations and opened two more units to offer alternative routes for patients: an AMAU and an SSU. The AMAU works as a 12-hour unit; it opens every weekday from 9:00 - 21:00, but only accepts patients up to 18:00, to allow beds to be freed for the next day. The SSU works as a short-stay ward for patients who need to be admitted to the hospital, and whose length of stay is estimated to be less than five days. The only access to the AMAU is through the ED after patients are assigned a triage category. The two units and the ED share resources, and also share resources with the hospital. In this study, a DES model is developed and tested to model the underlying relationships between the different units based on a previously developed model for the ED (Swallmeh et al. 2014).

\subsection{Data Collection and Analysis}

The qualitative and quantitative data used in this study were obtained from historical ED logs, electronic patient records (EPRs), direct observation and interviews. After the introduction of AMUs, the average patient experience time (PET) improved. While a medical patient spends an average of 9.06 hours in ED, another medical patient routed to the AMU spends an average of 4.11 hours from admission to discharge. Comparing the PET of medical patients in both units and the average time they spend in different care stages, it is clear that patients in the ED spend a significant amount of their time waiting to be moved into the department or waiting to be discharged or admitted. Also, a considerable number of patients who should be routed to the AMAU are misallocated to the ED, leading to an increase in their PET.

\subsection{Process Mapping}

On registration at the ED, walk-in patients remain in the waiting area to be triaged by a triage nurse. They are then assigned a triage category according to the Manchester Triage System (MTS), which is widely used in the UK, Europe generally, and Australia (Cronin 2003). Once assigned a triage category, a medical patient can be directed to either the ED or AMAU; the patient is eligible for the AMAU path if s/he arrives between 9:00 and 18:00 (the unit's admission hours) and is triaged in category 2 or 3 . If a patient is believed 
to be eligible for the AMAU path, the triage nurse calls the AMAU's consultant to inform him/her about the case and check the availability of a bed for the patient. If a bed is unavailable in the AMAU, the patient is referred to the ED path. Otherwise, the patient is directed to the AMAU and registers in their system. A nurse then interviews the patient and measures him/her vitals while waiting for a doctor's assessment. Most patients in the AMAU would follow the pathway in Figure 2, which shows the primary care steps relevant to all AMAU patients, whether they are discharged or admitted to the hospital.

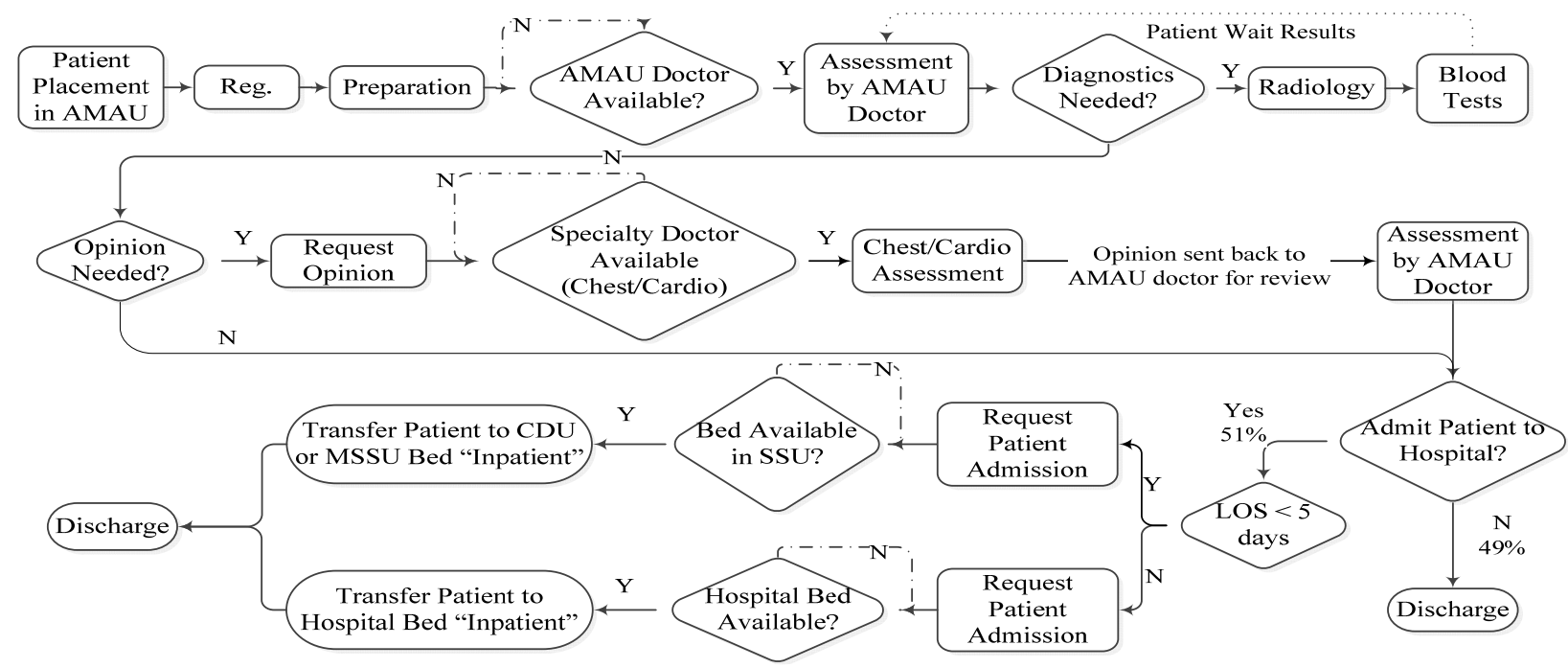

Figure 2: Patient's Flow in AMAU.

A conceptual model of the unit has been documented and validated by the hospital's senior clinicians and senior nursing staff. Based on this model and empirical data analysis, a comprehensive DES model has been developed. The simulation model was validated using face validation and comparison testing to ensure that the actual system length of stay times is mirrored by the model.

\subsection{Integrated Model}

The integrated model presented in this study is aimed at evaluating the AMU's intervention in Tallaght Hospital. A DES model was developed initially to provide better understanding of the interrelated dynamics between the system factors, and the responses that would give the clinicians insights into their system. It was also adopted to examine the impact of strategies proposed by the management team. Simulation outputs were able to identify all possible controllable and uncontrollable factors to be considered. The design of experiment (DoE) aims to measure the factors' impacts on five responses. Multivariate factor analysis then complemented the model by using the results of the DoE to ascertain the critical activities and determine the significant factors to each response. The DoE results were also used as an input to different machine learning techniques, used to find the best surrogate model to describe each response in terms of its predictors. A machine learning metamodel based on multi-objective simulation-optimization is proposed to support management decisions in identifying the bottleneck activities in the treatment journey of patients, and to determine the Pareto set of solutions to key decision variables, using simulated annealing (Figure 3).

\section{MULTIVARIATE FACTOR ANALYSIS}

Clinicians and executives were interested in identifying the most significant factors affecting the overall performance of the AMAU. Therefore, a DoE on the results of the simulation model was conducted. In practice, DoE is a useful tool, with many theoretical developments and practical applications in various fields. Latin hypercube sampling is a flexible way to build efficient designs for quantitative factors (Sanchez 
2005). Near-Orthogonal LH (NOLH) designs, specifically, have good space-filling and orthogonality properties with a small to an average number of factors (Cioppa and Lucas 2007).

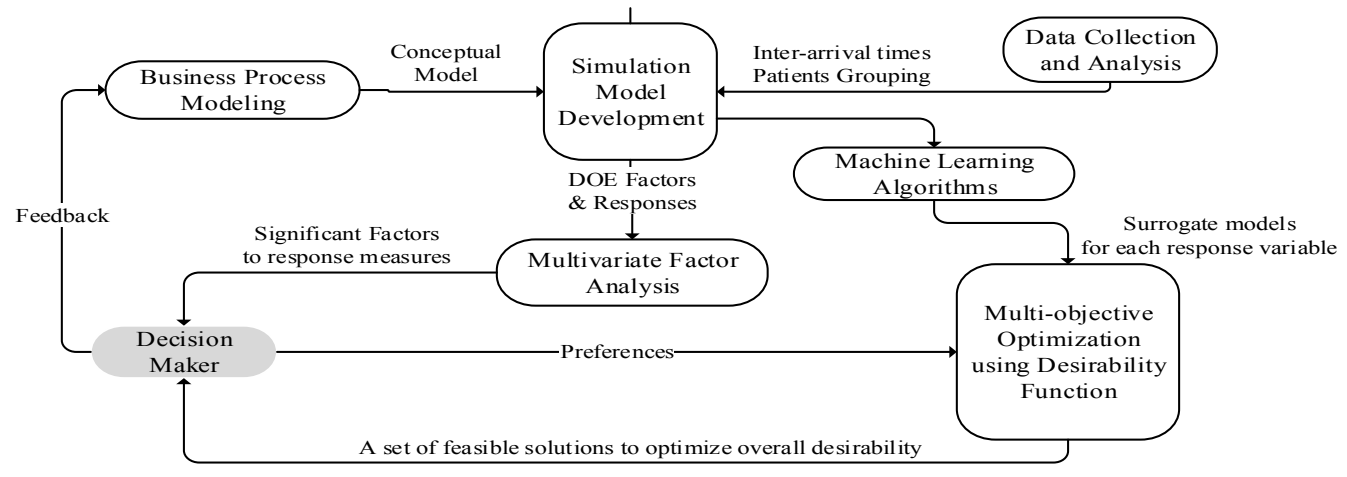

Figure 3: Different components of the proposed integrated model.

A NOLH design is employed in this study using 14 selected factors with 66 design points. This design is represented by a matrix $M_{n \times p}$ where $n$ is the number of scenarios (experiments), and $p$ is the number of factors (controllable and uncontrollable variables) that are uniformly spaced. Controllable variables are directly observable, such as the number of beds, whereas uncontrollable ones require statistical inference, such as patients' arrival rate (Kleijnen and Sargent 2000). The responses measured in each of these experiments (Table 1) are defined as the average patient's experience times in AMAU, the average patient's waiting time, the average number of patients accessing the AMAU, the average number of lost patients due to unavailable beds, and the average of boarded patients in the AMAU due to unavailable downstream beds (wards and SSU). The response matrix is evaluated using the simulation model for each experiment.

Table 1: Description of DOE variables and responses.

\begin{tabular}{|c|c|c|}
\hline \multicolumn{2}{|r|}{ Factors/Predictors } & \multirow{2}{*}{ Levels } \\
\hline Type & Variable Description & \\
\hline 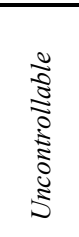 & $\begin{array}{l}\boldsymbol{p}_{1}: \text { Patients' misallocation. } \\
\boldsymbol{p}_{2}: \text { Opening Hours }(12 \mathrm{hrs}, 18 \mathrm{hrs}, 24 \mathrm{hrs} \text {.) } \\
\boldsymbol{p}_{3}: \text { Service time change } \\
\boldsymbol{p}_{4}: \text { Inter-arrival time at night in minutes } \\
\boldsymbol{p}_{5}: \text { Inter-arrival time evening in minutes } \\
\boldsymbol{p}_{6}: \text { Inter-arrival time morning in minutes } \\
\boldsymbol{p}_{7}: \text { Average number of ED boarders }\end{array}$ & $\begin{array}{l}\text { 2L: } 0 \text { or } 1 \\
\text { 3L: }-1,0 \text { and }+1 \\
\text { 3L: }-10 \%, 0,10 \% \\
\text { 3L: } 27,30 \text { and } 33 \\
\text { 3L: } 11,12 \text { and } 13 \\
\text { 3L: } 7,8 \text { and } 9 \\
\text { 8L: } 0,1,2 \ldots 7\end{array}$ \\
\hline $\begin{array}{l}\frac{0}{0} \\
\text { రే } \\
0 \\
0 \\
0\end{array}$ & $\begin{array}{l}\text { 1: Number of AMAU beds } \\
\text { x2: Number of SSU beds } \\
\text { x3: Number of ward beds } \\
\text { x4: Number of Nurses } \\
\text { x5: Number of Registrars } \\
\text { x6: Number of SHOs } \\
\text { x7: } \text { Number of Consultants }\end{array}$ & $\begin{array}{l}8 \mathrm{~L}: 9,10 \ldots 16 \\
16 \mathrm{~L}: 9,10 \ldots 24 \\
66 \mathrm{~L}: 470 \text { to } 570 \\
3 \mathrm{~L}: 2,3 \text {, and } 4 \\
3 \mathrm{~L}: 1,2 \text {, and } 3 \\
3 \mathrm{~L}: 1,1 \text {, and } 1 \\
\text { 2L: } 1 \text { and } 2\end{array}$ \\
\hline
\end{tabular}

\begin{tabular}{|l|}
\hline \multicolumn{1}{|c|}{ Responses Variables } \\
\hline $\boldsymbol{y}_{1}:$ Average patient's experience time (PET) in \\
AMAU \\
$\boldsymbol{y}_{2}:$ Average patient's waiting time in AMAU \\
$\boldsymbol{y}_{3}:$ AMAU accessibility; average number of daily \\
patients accessing AMAU \\
$\boldsymbol{y}_{4}:$ AMAU blocking; the average number of lost \\
patients daily due to bed unavailability. \\
$\boldsymbol{y}_{5}:$ AMAU Boarders; average number of boarded \\
patients daily in AMAU due to unavailability of \\
downstream resources (i.e. SSU and ward beds) \\
\\
\end{tabular}

\subsection{Performance Determinants}

From a practical perspective, a transformation of the predictor and response variables is required before analysis, due to the different scales of the predictors and also where some of them are found to be skewed. The most frequent and straightforward transformation is to centre and scale the predictors. It is possible to centre the data by subtracting the sample mean from all the values while scaling the data requires each value 


\section{Rashwan, Habib, Arisha, Courtney, and Kennelly}

of a variable to be divided by its sample standard deviation. In addition to centring and scaling, removing the distributional skewness is a major step in improving the numerical stability of the calculations. The skewness of the variables is fixed using Yeo-Johnson Power Transformations (Yeo and Johnson 2000).

The variables were examined separately for the 66 design points. The transformed factor and response matrix are $x^{*}$ and $y^{*}$ respectively. Standard multiple regression was performed between each response variable separately as a dependent variable and with $x^{*}$ as the independent variables. Table 2 displays the standardized/transformed regression coefficients, intercept, significance, standard error and $\mathrm{R}^{2}$. The $\mathrm{R}^{2}$ for all regression models is significantly different from zero, at $p<0.001$.

Table 2: Regression analysis results.

\begin{tabular}{|c|c|c|c|c|c|}
\hline \multicolumn{6}{|c|}{ Analysis of Deviance Table (Type II tests) } \\
\hline \multirow{4}{*}{ Factors } & \multicolumn{5}{|c|}{\begin{tabular}{|c|} 
Responses \\
\end{tabular}} \\
\hline & PET, $y_{1}$ & Waiting Time, $y_{2}$ & AMAU Access, $y_{3}$ & Blocked Patients, $y_{4}$ & Internal Boarders, $y_{5}$ \\
\hline & Estimate (Std. Error) & Estimate (Std. Error) & Estimate (Std. Error) & Estimate (Std. Error) & Estimate (Std. Error) \\
\hline & $-1.2811(0.1198)^{* * *}$ & $-1.2218(0.1543)^{* * *}$ & $-1.1909(0.1199)^{* * *}$ & $1.1773(0.1147)^{* * *}$ & $0.4381(0.2112)^{*}$ \\
\hline$p_{1}:$ Misallocation & $0.5882(0.1161)^{* * *}$ & $0.3874(0.1496)^{*}$ & $0.3467(0.1162)^{* * *}$ & $-0.3662(0.1111)^{* *}$ & $0.407(0.2047)^{\wedge}$ \\
\hline$p_{2}$ : Opening Hours & $0.987(0.0797) * * *$ & $1.0281(0.1026) * * *$ & $1.0175(0.0797)^{* * *}$ & $-0.9942(0.0762) * * *$ & $-0.6416(0.1404) * * *$ \\
\hline$p_{3}:$ Service Time & $0.1791(0.0569) * *$ & $-0.108(0.0733)$ & $0.0353(0.0569)$ & $-0.0309(0.0545)$ & $0.004(0.1003)$ \\
\hline$p_{4}$ : Inter-arrival time1 & $0.0308(0.0587)$ & $-0.003(0.0756)$ & $-0.0098(0.0587)$ & $-0.0725(0.0562)$ & $0.0319(0.1034)$ \\
\hline ps: Inter-arrival time2 & $-0.0748(0.0573)$ & $0.0371(0.0738)$ & $-0.1009(0.0573)$ & $-0.1428(0.0548) *$ & $-0.0397(0.1009)$ \\
\hline$p_{6}$ : Inter-arrival time3 & $0.066(0.0582)$ & $-0.0076(0.0749)$ & $-0.0089(0.0582)^{\wedge}$ & $-0.3742(0.0557) * * *$ & $0.0141(0.1025)$ \\
\hline$p_{7:}$ ED Boarders & $-0.2269(0.0566)^{* * * *}$ & $-0.1819(0.0729) *$ & $-01868(0.057)^{* * *}$ & $0.19(0.0542) * * *$ & $-0.1667(0.0998)^{\wedge}$ \\
\hline$x_{1}:$ AMAU Beds & $0.0505(0.0576)$ & $-0.0818(0.0742)$ & $0.0634(0.0576)$ & $-0.0505(0.0551)$ & $0.1142(0.1015)$ \\
\hline$x_{2}:$ SSU Beds & $-0.2229(0.0572) * * *$ & $-0.3134(0.0737) * * *$ & $0.1482(0.0572) *$ & $-0.1519(0.0548) * *$ & $-0.2039(0.1008) *$ \\
\hline$x_{3}:$ Ward Beds & $-0.0839(0.0566)$ & $-0.1932(0.0729) *$ & $0.3999(0.0566) * * *$ & $-0.3554(0.0542)^{* * *}$ & $-0.3632(0.0998) * * *$ \\
\hline$x_{4}:$ Nurses & $0.0571(0.0576)$ & $0.0677(0.0742)$ & $-0.0541(0.0576)$ & $0.048(0.0551)$ & $-0.0125(0.1015)$ \\
\hline$x_{5}:$ Registrar & $-0.269(0.0568) * * *$ & $-0.1672(0.0731) *$ & $0.0179(0.0568)$ & $-0.0181(0.0543)$ & $-0.025(0.1)$ \\
\hline$x_{6}: \mathrm{SHO}$ & $-0.1699(0.0583)^{* *}$ & $-0.1185(0.075)$ & $0.0697(0.0583)$ & $-0.0482(0.0557)$ & $0.0271(0.1027)$ \\
\hline$x_{7}:$ Consultant & $-0.3311(0.0575)^{* * *}$ & $-0.1174(0.0741)$ & $0.0052(0.0575)$ & $-0.0106(0.055)$ & $-0.0536(0.1013)$ \\
\hline
\end{tabular}

- Patient Experience Time - Eight variables, $x_{1}, x_{2}, x_{3}, x_{5}, x_{8}, x_{9}, x_{10}$ and $x_{14}$, contributed significantly to PET in AMAU $\left(y_{1}\right)$, at $p<0.001$. Altogether, $84.26 \%$ of the variability in the AMAU PET was predicted based on these eight variables. Misallocation $x_{1}$, opening hours $x_{2}$ and service time $x_{3}$ are positively associated with this response. It is evident that increasing the demand and service time of the unit would lead to an increase in the time experienced by the patient. On the other hand, $y_{l}$ is negatively associated with increasing staff capacity $x_{8}, x_{9}$ and $x_{10}$, and the downstream bed capacity (SSU bed; $x_{5}$ ). Also, increasing the number of ED boarders has a negative impact on the AMAU PET because the boarded patients block admission to the unit, which indirectly reduces the average PET of the admitted patients. Counter-intuitively, the unit bed capacity, $x_{4}$, has an insignificant impact on the PET, which may explain why the AMAU beds are not a capacity constraint (a bottleneck). The size of the relationship suggests that opening hours and misallocation have the highest positive impact while the number of consultants and registrars has the largest negative impact.

- Waiting Time - The variables $x_{1}, x_{2}, x_{5}, x_{6}, x_{8}$ and $x_{14}$ were identified as having a significant impact on waiting times in AMAU $\left(y_{2}\right), p<0.001$. The predictors can explain $73.7 \%$ of the total variation in the average waiting time. The results reflect a strong positive association between demand factors $x_{1}$, and $x_{2}$ while waiting time on the downstream resources, $x_{5}$ and $x_{6}$, the number of registrars, $x_{8}$, and ED boarders, $x_{14}$, are negatively correlated with average waiting time in AMAU, $y_{2}$.

- The AMAU accessibility - Six independent variables, $x_{1}, x_{2}, x_{5}, x_{6}, x_{13}$, and $x_{14}$ have a significant impact on the patient's accessibility to the AMAU unit, $y_{3}$, at $p<0.0001$. These predictors can explain $84 \%$ of the total variance in the number of patients admitted to the AMAU unit. More available downstream beds, $x_{5}$ and $x_{6}$, would lead to more admissions to the AMAU. Also, increasing the number of patients arrival during the AMAU opening hours, $x_{13}$, and increasing the 
demand, $x_{1}$, and $x_{2}$, were associated with increasing the number of admitted patients to the AMAU. Apparently, the ED boarded patients in the AMAU, $x_{14}$, contribute negatively to the acceptance of more patients. Also, the results showed that the AMAU bed capacity, $x_{4}$, has no significant effect on admissions as the unit is constrained by resources in the subsequent stages.

- Blocked Patients - This response summarizes the number of blocked patients due to opening hours, misallocation, bed unavailability, boarding and reneging. Seven predictors were defined that have a significant effect on this response: $x_{1}, x_{2}, x_{5}, x_{6}, x_{12}, x_{13}$, and $x_{14}$. The first six variables showed a negative relationship with the number of blocked patients in the unit. Also, the number of boarders from the ED showed a positive correlation with the number of blocked patients. Hence, a control on how many patients are allowed to board the unit's beds would lead to an improvement in this figure.

- AMAU Boarding - The multiple linear regression analysis was conducted to predict the AMAU boarded patients, $y_{6}$, based on all factor variables. A significant relationship was found $(p<0.0001)$, with $R^{2}$ of $51.1 \%$. Five variables, $x_{1}, x_{2}, x_{5}, x_{6}$, and $x_{14}$, have contributed significantly to predicting a number of AMAU's boarders due to the unavailability of beds in the SSU or ward.

In the current individual setting of the AMAU under study, the AMAU's bed capacity did not have a significant impact on any of the performance variables, which shows that this factor should not be presented as a bottleneck.

\section{MULTI-OBJECTIVE OPTIMIZATION}

Focusing only on the strongest predictors of response measures from the regression analysis may not always be the most effective when planning for improvement strategies. The ultimate objective of this study was to find the optimal configuration of the main unit resources in order to optimize multiple objectives simultaneously, subject to a set of capacity constraints. The mathematical formulation of the problem (P) is:

$$
\begin{gathered}
\operatorname{Min} Y=\left\{f_{1}(x), f_{2}(x),-f_{3}(x), f_{4}(x), f_{5}(x)\right\} \\
\text { s.t } \\
\quad l \leq \mathrm{x} \leq u \text { and } \mathrm{x} \in \text { Integer set. }
\end{gathered}
$$

The objectives are to minimize the average PET in the AMAU, $f_{1}(x)$, average waiting time, $f_{2}(x)$, average number of blocked patients, $f_{4}(x)$, and the average number of boarded patients, $f_{5}(x)$, while maximizing the mean access to the unit, $f_{3}(x)$. The vectors $l$ and $u$ are the lower and upper capacity levels of the decision variables $x$ based on the decision-maker's recommendations. Since this multi-objective discrete optimization problem doesn't have a closed analytical form; it should be evaluated using the simulation model, where $y_{i}(x)$ is the estimation of $f_{i}(x)$.

\subsection{Machine Learning (ML) based Surrogate-Models}

A set of supervised ML predictive models is tested to select the best surrogate model for each response variable. Three groups of predictive models are tested for each response variable. The first group is the linear regression models, which include ordinary least squares regression (OLS), partial least squares (PLS), elastic net, and linear support vector machines (Linear SVM). The second group is the non-linear retrogression models. The nonlinear regression models tested are neural networks (Nnet), multivariate adaptive regression splines (MARS), and radial basis function SVM (Radial SVM). Finally, regression tree and rule-based models are tested, consisting of a set of nesting if - then rules for the predictors that partition the data. Six regression trees and rule-based models are examined for each response variable: classification and regression tree (CART), condition inference tree, bagged tree, boosted tree and random forest $(R F)$ and Cubist. To reducing the noise, five replications are employed in this study, resulting in an acceptable level of mean square error. The training dataset for each ML model is obtained from the NOLH discussed earlier. Typically, the Root Mean Square Error (RMSE) is used to evaluate the effectiveness of 
the regression models. RMSE is employed to assess the predictive performance of the ML models (next section).

\subsection{Parameter Tuning and Model Selection}

For each response variable, 13 predictive ML models were assessed over a defined set of tuned parameters (grid search). The performance metric was evaluated using five repeats of 10-fold cross-validation. In the process of parameter tuning, cross-validation generated 50 different estimates of the accuracy of each candidate of model parameters. A resampling method is used to compare between these 13 models, based on the cross-validation statistics. Table 3 summarizes the RMSE calculated from the cross-validation of each response variable across all models. Since the models' accuracies are evaluated using identically resampled data, the paired-t test can be applied to compare the significant differences among these models for each response separately.

The main criterion for selecting the appropriate surrogate model is the RMSE (Table 3). However, when there is no significant difference between the surrogate models (using paired-t test), the most interpretable model is selected as a rule of thumb. The rule of thumb for the results indicated that LSVM has the best cross-validation accuracy for $\widehat{y}_{1}$, but there is no evidence - with a $95 \%$ confidence level - to support the idea that the accuracy of LSVM is better than that of OLS, PLS, elastic net, MARS, or Cubist. In this case, the elastic net model is selected as a candidate metamodel to predict the PET in AMAU ( $\left.\hat{\mathrm{y}}_{1}\right)$ regarding the decision variables, because it is a less opaque model compared with LSVM. The MARS and Cubist models outperformed the rest of the models for predicting the waiting time $\left(\hat{y}_{2}\right)$, access to AMAU $\left(\hat{y}_{3}\right)$, number of blocked patients $\left(\hat{y}_{4}\right)$ and number of boarded patients $\left(\hat{y}_{5}\right)$; the paired t-test showed that there is no significant difference in the accuracy between these two models; therefore MARS was the selected model to predict the responses, because it is more interpretable than the Cubist model. The elastic net has two tuned parameters (fraction, $\lambda$ ) and MARS also has two parameters (no. of prune, degree). The values of the tuned parameters used for each selected model are presented in the last column of Table 3.

Table 3: Machine learning models for selecting the appropriate surrogate models.

\begin{tabular}{|c|c|c|c|c|c|c|c|c|c|c|c|c|c|c|c|}
\hline \multirow{2}{*}{$\begin{array}{l}\text { Resp. } \\
\text { Var. }\end{array}$} & \multicolumn{4}{|c|}{ Linear Regression Models } & \multicolumn{3}{|c|}{$\begin{array}{c}\text { Nonlinear } \\
\text { Regression Models }\end{array}$} & \multicolumn{6}{|c|}{ Tree and Rule-based Regression Models } & \multirow{2}{*}{$\begin{array}{c}\text { Selected } \\
\text { Model }\end{array}$} & \multirow{2}{*}{$\begin{array}{c}\text { Tuned } \\
\text { Parameters }\end{array}$} \\
\hline & OLS & PLS & $\begin{array}{c}\text { Elastic } \\
\text { Net }\end{array}$ & LSVM & MARS & RSVM & Nnet & CART & $\begin{array}{l}\text { Cond Inf } \\
\text { Tree }\end{array}$ & $\begin{array}{c}\text { Bagged } \\
\text { Tree }\end{array}$ & $\begin{array}{c}\text { Boosted } \\
\text { Tree }\end{array}$ & $\begin{array}{c}\text { Radom } \\
\text { Forest }\end{array}$ & Cubist & & \\
\hline$\widehat{y}_{1}$ & $0.65^{*}$ & $0.64 *$ & $0.64 *$ & $0.62 *$ & $0.66^{*}$ & 0.71 & 0.71 & 0.94 & 0.94 & 0.89 & 0.66 & 0.86 & $0.63 *$ & Elastic net & $(.9, .01)$ \\
\hline$\widehat{y}_{2}$ & 2.50 & 2.36 & 2.11 & 2.39 & $1.23 *$ & 2.25 & 2.29 & 1.86 & 1.86 & 1.85 & 1.83 & 1.79 & $1.25^{*}$ & MARS & $(4,2)$ \\
\hline$\widehat{y}_{3}$ & 8.02 & 7.87 & 7.37 & 8.76 & 4.87* & 7.48 & 7.35 & 7.86 & 7.92 & 8.16 & 5.47 & 6.33 & $4.97 *$ & MARS & $(7,1)$ \\
\hline$\widehat{y}_{4}$ & 14.28 & 14.20 & 13.89 & 15.35 & $6.51 *$ & 14.87 & 9.80 & 14.42 & 14.42 & 14.62 & 9.69 & 9.64 & $7.40 *$ & MARS & $(12,2)$ \\
\hline$\widehat{y}_{5}$ & 4.72 & 4.60 & 4.27 & 4.67 & $2.80 *$ & 4.11 & 4.84 & 4.48 & 4.43 & 3.97 & 3.31 & 3.27 & $2.18 *$ & MARS & $(15,3)$ \\
\hline
\end{tabular}

* There is no significant difference between these models based on a paired-t test at $95 \%$ confidence level for each solution.

\subsection{Desirability-Based Multi-Objective}

The desirability function approach was introduced by Harrington (1965) to optimize multiple simultaneous objectives by transforming the estimated response $\widehat{y}_{l}(\mathrm{x})$ into a unified scale $[0,1]$, called a desirability index, denoted by $d_{i}\left(\widehat{y}_{l}(\mathrm{x})\right)$. The highest desirable solution has a value of 1 while a highly undesirable solution has a value of 0 . The desirability function for minimization and maximization-type response functions are given by equations (1) and (2) respectively:

$$
d_{i}^{\text {min }}\left(\widehat{y}_{l}(\mathrm{X})\right)=\left\{\begin{array}{cl}
\left(\frac{\widehat{y}_{l}(\mathrm{X})-Y_{i}^{\text {min }}}{Y_{i}^{\text {max }}-Y_{i}^{\text {min }}}\right)^{\delta_{i}} & \text { if } \left.{\widehat{\widehat{y}_{l}}} \mathrm{X}\right)>Y_{i}^{\text {max }} \\
1 & \text { if } Y_{i}^{\text {min }} \leq \widehat{y}_{l}(\mathrm{X}) \leq Y_{i}^{\text {max }} \\
\text { if } \widehat{y}_{l}(\mathrm{X})<Y_{i}^{\text {mminax }}
\end{array}\right.
$$


Rashwan, Habib, Arisha, Courtney, and Kennelly

$$
d_{i}^{\text {max }}\left(\widehat{y}_{l}(\mathrm{X})\right)=\left\{\begin{array}{cl}
0 & \text { if } \widehat{y}_{l}(\mathrm{X})<Y_{i}^{\text {min }} \\
\left(\frac{\widehat{y}_{l}(\mathrm{X})-Y_{i}^{\text {max }}}{Y_{i}^{\text {min }}-Y_{i}^{\text {max }}}\right)^{\delta_{i}} & \text { if } Y_{i}^{\text {min }} \leq \widehat{y}_{l}(\mathrm{X}) \leq Y_{i}^{\text {max }} \\
1 & \text { if } \left.{\widehat{y_{l}}}_{l} \mathrm{X}\right)>Y_{i}^{\text {max }}
\end{array}\right.
$$

$Y_{i}^{\min }$ and $Y_{i}^{\max }$ are the lower and upper bound of the response $\widehat{y}_{l}$ respectively, and $\delta_{i}$ is the shape parameter of the desirability function $d_{i}\left(\widehat{y}_{l}(\mathrm{x})\right)$. The desirability function is linear if $\delta_{i}=1$ and convex (concave) if $\delta_{i}>1\left(\delta_{i}<1\right)$. The shape parameter is chosen such that the desirability is easier or more difficult to achieve. $Y_{i}^{\min }$ and $Y_{i}^{\max }$ are calculated using the quantile ranks. These parameters are chosen by the modeller in coordination with the decision-maker.

\section{$5.4 \quad$ Optimization Results}

A composite or overall desirability $(D)$ of a solution (system configuration) combines all the individual desirability values $d_{i}\left(\widehat{y}_{l}(\mathrm{x})\right)$ using the geometric mean. Derringer (1994) proposed a weighted geometric mean, which is given by $D=\prod_{i}^{p} d_{i}\left(\hat{y}_{i}\right)$. Replacing the multiple objectives with the composite desirability function $D$ obtains a new single-objective to be used to find the set of efficient solutions. Gradient-based search methods are not suitable for this problem since the various prediction models are not smooth and have many discontinuities (e.g. MARS and Cubist). A direct search method is applied to obtain a nearoptimal configuration: simulated annealing (SA) (Suman and Kumar 2006). SA is a global search technique that attempts to avoid the possibility of being trapped in local optima through accepting poor solutions. The acceptance/rejection of worse solutions is controlled by a probability function that depends on a temperature parameter to trade off exploration and exploitation.

The search is repeated several times using different starting points to overcome being stuck in local optima, and also to generate a set of solutions from which the best can be selected. A total of 15 starting points are chosen from the DoE dataset; the first starting point of the 15 is sampled randomly, and the rest are chosen using the maximum dissimilarity sampling method. All constraints are handled using the penalty function. Table 4 presents the results obtained from the SA method; it is divided into four sections: values of decision variables, the corresponding responses, the responses' desirability, and the composite desirability of the solution. The first row shows the current solution; each following row presents a different solution obtained from the 15 starting points. Out of the 15 starting points, only seven trails succeeded in reaching a feasible solution.

Table 4: A set of efficient solutions obtained from SA method with multiple starts.

\begin{tabular}{|c|c|c|c|c|c|c|c|c|c|c|c|c|c|c|c|c|}
\hline \multirow[t]{2}{*}{ Solutions } & \multicolumn{7}{|c|}{ Decision variables } & \multicolumn{3}{|c|}{ Responses } & \multicolumn{5}{|c|}{ Individual Desirability } & \multirow{2}{*}{\begin{tabular}{|c} 
Overall \\
$D$
\end{tabular}} \\
\hline & $x_{1}$ & $x_{2}$ & $x_{3}$ & $x_{4}$ & $x_{5}$ & $x_{6}$ & $x_{7}$ & $\widehat{y}_{1}$ & $\begin{array}{lll}\widehat{y}_{2} & \widehat{y}_{3} & \widehat{y}_{4} \\
\end{array}$ & $\widehat{\boldsymbol{y}}_{\mathbf{5}}$ & $d_{1}$ & $d_{2}$ & $d_{3}$ & $d_{4}$ & $d_{5}$ & \\
\hline Current & 11 & 12 & 520 & 3 & 2 & 2 & 1 & 4.125 & 1.54120 .7901 .358 & 1.849 & 0.884 & 0.812 & 0.807 & 1.0 & 0.744 & 0.845 \\
\hline Solution 1 & 16 & 16 & 510 & 2 & 2 & 2 & 2 & 3.460 & 0.83021 .6861 .226 & 1.210 & 1.00 & 1.000 & 0.835 & 1.000 & 0.942 & 0.953 \\
\hline Solution 2 & 10 & 15 & 498 & 3 & 1 & 2 & 2 & 3.872 & 1.15117 .1633 .998 & 2.225 & 0.95 & 0.946 & 0.650 & 0.993 & 0.634 & 0.819 \\
\hline Solution 3 & 11 & 15 & 544 & 2 & 2 & 3 & 2 & 3.235 & 0.61923 .1340 .068 & 0.922 & 1.00 & 1.000 & 0.874 & 1.000 & 1.000 & 0.973 \\
\hline Solution 4 & 11 & 16 & 519 & 2 & 3 & 2 & 2 & 3.110 & 0.53323 .1421 .352 & 0.154 & 1.00 & 1.000 & 0.874 & 1.000 & 1.000 & 0.973 \\
\hline Solution 5 & 11 & 14 & 496 & 3 & 2 & 3 & 2 & 3.425 & 1.07915 .9704 .272 & 3.024 & 1.00 & 0.970 & 0.558 & 0.975 & 0.422 & 0.741 \\
\hline Solution 6 & 11 & 11 & 491 & 2 & 1 & 3 & 1 & 4.697 & 2.11514 .7545 .890 & 5.110 & 0.719 & 0.580 & 0.347 & 0.868 & 0.034 & 0.335 \\
\hline Solution 7 & 13 & 15 & 530 & 2 & 3 & 1 & 2 & 3.337 & 0.57122 .4791 .149 & 0.184 & 1.00 & 1.000 & 0.857 & 1.000 & 1.000 & 0.970 \\
\hline
\end{tabular}

Note: The rejected (dominated) solutions are struck-through.

The current solution is strongly dominated by solutions 1, 3 and 7. Also, solutions 5 and 6 are local optima points regarding the value of their composite desirability $D$ since all other solutions dominate them; therefore, they can be safely excluded. Solutions 1 and 2 can also be excluded since they are strongly dominated by solution 3 . The non-dominated solution set is formed from solutions 3, 4 and 7; comparing their desirability values, solution 3 and 4 weakly dominate solution 7. Also, comparing the input/output 
levels of each non-dominated solution reveals that solution 4 is the best configuration. This configuration improves the patient-related indicators significantly with the minimum extra resources. This solution suggests the need to increase the bottleneck resources that limit the patient flow.

The results of comparing the current AMAU settings and the best (near optimal) setting, solution 4, (Figure 4) show some improvement in all the response variables. The horizontal line represents the current performance (as 100\%), and the bar chart shows the response variables of the best setting as a percentage of the current setting. The average PET, average waiting time and number of AMAU boarders are dramatically decreased - by $25 \%$ and $65 \%$ respectively. The AMAU boarders are nearly eliminated, while AMAU accessibility (patient flow) has increased by around $11 \%$. It is likely that the expected performance improvements in the optimal scenario would be subject to the implementation challenges.

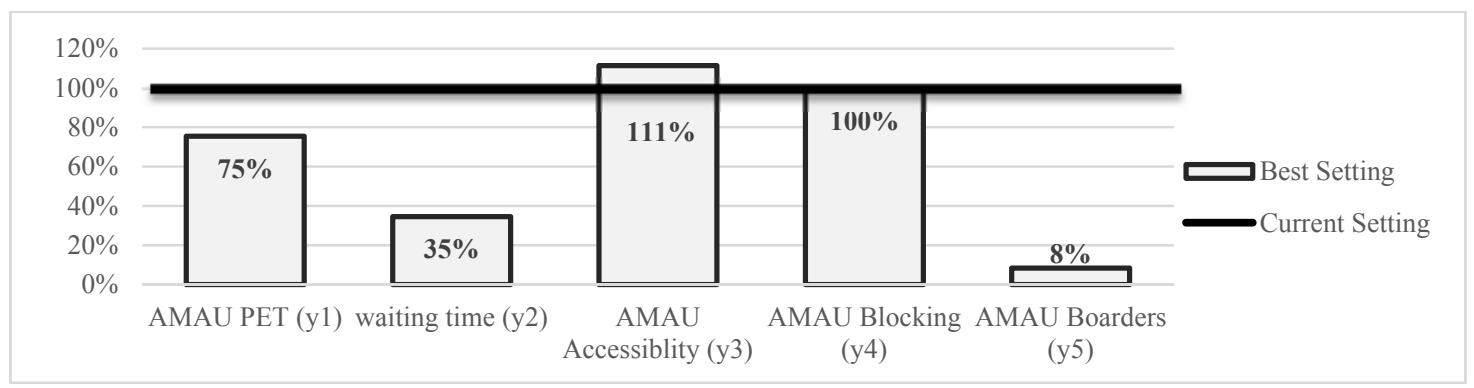

Figure 4: Comparison between current and the best scenario setting of the AMAU.

\section{CONCLUSION}

With the increasing demand for emergency services, Emergency Departments (EDs) are starving for support. Hospitals desiring to improve patients' experience have to implement facilitative interventions. The Acute Medicine Program (AMP) provides a framework to deliver acute medical services aimed at substantially improving patient care, reducing waiting times in emergency units, and helping to shorten the decision-making process in a safe way. It proposes that specialized units known as Acute Medical Assessment Units (AMAUs) work in parallel with EDs to alleviate the pressure caused by high demand. The main contribution of this study is to introduce an integrated model that is used to evaluate the integration of AMAU in Tallaght Hospital, Dublin.

A Discrete Event Simulation (DES) model was initially developed to provide insights and understand the dynamics of the selected units in the hospital. Several experiments proposed by the management team were studied; the simulation model was used to examine the impact of different strategies on the AMAU's performance. Fourteen factors were identified from the simulation results as being significant to the response variables suggested by the unit's management. These factors and patient-related responses were then used to develop a Design of Experiments (DoE) with good space-filling to cover all possible outcomes of the system. Sixty-six different scenarios resulting from the design were evaluated by the simulation model; they were then used in performing a multivariate factor analysis to identify the performance determinants for each response variable.

Results from the factor analysis showed that downstream capacities had significant impacts on all performance variables. Therefore, their exact values needed to be optimized to help alleviate the pressure on the unit in a cost-effective manner. Similarly, patient misallocation, ED boarded patients and the unit's opening hours considerably affected all the unit responses. However, in the individual setting of the AMAU under study, and at variance with common clinician perceptions, the AMAU's bed capacity did not have a major impact on any of the performance variables, indicating that increasing bed capacity will not alleviate pressure on the unit. The DoE results were also used as inputs to exploit 13 machine learning predictive metamodels in an attempt to provide surrogate models for each response variable. Surrogate models were 
employed to describe the relationships between response variables and their predictors and then used in the multi-objective optimization model since there is no closed analytical form to predict the responses. The literature indicates that machine learning algorithms are effective in attaining a closed form for each response variable. The results show that multivariate adaptive regression splines (MARS) outperformed most other methods for predicting the surrogate model for all response variables, except for the average patient experience time (PET) in AMAU, where elastic nets had the best root mean square error.

Finally, the model proposed provides real-time strategies for AMAUs to improve patient care, by introducing a multi-objective optimization approach that aims to compromise among various objectives. The desirability function method is used to convert the multiple objectives into a single objective problem. The simulated annealing (SA) approach is then used to obtain near-to-optimal solutions. The search is repeated 15 times, with different starting points to avoid being trapped in local minima. The suggested solutions from the SA show that developing downstream capacity along with increasing the unit's human resources would lead to a 25\% decrease in PET and significantly improve other response variables, allowing the AMAU to meet the unpredictable increase in demand better.

\section{REFERENCES}

Barton, R., and M. Meckesheimer. 2006. "Metamodel-Based Simulation Optimization." Handbooks in Operations Research and Management Science 13 (C): 535-574.

Bond, K., M. Ospina, S. Blitz, M. Afilalo, S. Campbell, M. Bullard, G. Innes, B. Holroyd, G. Curry, M. Schull, and B. H. Rowe. 2007. "Frequency, Determinants and Impact of Overcrowding in Emergency Departments in Canada: A National Survey." Healthcare Quarterly 10: 32-40.

Brailsford, S. 2006. "Overcoming the Barriers to Implementation of Operations Research Simulation Models in Healthcare." Clinical and Investigative Medicine 28 (6): 312-315.

Cioppa, T., and T. Lucas. 2007. "Efficient Nearly Orthogonal and Space-Filling Latin Hypercubes." Technometrics 49 (1): 45-55.

Cronin, J. G. 2003. "The Introduction of the Manchester Triage Scale to an Emergency Department in the Republic of Ireland." Accident and Emergency Nursing 11 (2): 121-125.

Davies, R. 2007. "'See and Treat' or 'See' and 'Treat' in an Emergency Department." In Proceedings of the 2007 Winter Simulation Conference, edited by S. G. Henderson, B. Biller, M. H. Hsieh, J. Shortle, J. D. Tew, and R. R. Barton, 1519-22. Piscataway, New Jersey: Institute of Electrical and Electronics Engineers, Inc.

Dellino, G., J. Kleijnen, and C. Meloni. 2009. "Robust Simulation-Optimization Using Metamodels." In Proceedings of the 2009 Winter Simulation Conference, edited by M. D. Rossetti, R. R. Hill, B. Johansson, A. Dunkin, and R. G. Ingalls, 540-550. Piscataway, New Jersey: Institute of Electrical and Electronics Engineers, Inc.

Derringer, G. 1994. “A Balancing Act : Optimizing a Product's Properties.” Quality Progress 27(6):51-58.

Forero, R., K. Hillman, S. McCarthy, D. Fatovich, A. Joseph, and D. Richardson. 2010. "Access Block and ED Overcrowding: Short Report.” EMA - Emergency Medicine Australasia 22 (2): 119-135.

Graff, L. 1999. "Overcrowding in the ED: An International Symptom of Health Care System Failure." American Journal of Emergency Medicine 17 (2): 208-209.

Harper, P. 2002. "A Framework for Operational Modelling of Hospital Resources." Health Care Management Science 5 (3): 165-73.

Harrington, E. C. 1965. “The Desirability Function.” Industrial Quality Control 21 (10): 494-498.

HSE. 2010. "Report of the National Acute Medicine Programme." http://www.hse.ie/eng/about/Who/clinical/natclinprog/acutemedicineprogramme/report.pdf.

HSE. 2015. "November Performance Rort." https:/www.hse.ie/eng/services/publications/performancereports/novpr.pdf.

Kleijnen, J. 2005. "An Overview of the Design and Analysis of Simulation Experiments for Sensitivity 
Analysis.” European Journal of Operational Research 164 (2): 287-300.

Kleijnen, J., and R. Sargent. 2000. "A Methodology for Fitting and Validating Metamodels in Simulation.” European Journal of Operational Research 120: 14-29.

Oddoye, P., F. Jones, M. Tamiz, and P. Schmidt. 2009. "Combining Simulation and Goal Programming for Healthcare Planning in a Medical Assessment Unit." European Journal of Operational Research 193 (1): 250-61.

Poropudas, J., and K. Virtanen. 2011. "Simulation Metamodeling with Dynamic Bayesian Networks." European Journal of Operational Research 214 (3). Elsevier B.V.: 644-655.

Reis dos Santos, P., and M. I. Reis dos Santos. 2009. "Using Subsystem Linear Regression Metamodels in Stochastic Simulation.” European Journal of Operational Research 196 (3): 1031-1040.

Sanchez, S. M. 2005. "Guidelines for Designing Simulation Experiments." In Proceedings of the 2006 Winter Simulation Conference, edited byL. F. Perrone, F. P. Wieland, J. Liu, B. G. Lawson, D. M. Nicol, and R. M. Fujimoto, 47-57. Piscataway, New Jersey: IEEE, Inc.

Schafermeyer, R., and B. Asplin. 2003. "Hospital and Emergency Department Crowding in the United States." Emergency Medicine 15 (1): 22-27.

Suman, B, and P Kumar. 2006. "A Survey of Simulated Annealing as a Tool for Single and Multiobjective Optimization." Journal of the Operational Research Society 57 (10): 1143-1160.

Swallmeh, E., A. Tobail, W. Abo-hamad, J. Gray, and A. Arisha. 2014. "Integrating Simulation Modelling and Value Stream Mapping for Leaner Capacity Planning of an Emergency Department." In The Sixth International Conference on Advances in System Simulation, 256-262.

Thorwarth, M., W. Rashwan, and A. Arisha. 2015. "An Analytical Representation of Flexible Resource Allocation in Hospitals.” Flexible Services and Manufacturing Journal 26 (93): 1989-2014.

Yeo, I. K., and R. Johnson. 2000. "A New Family of Power Transformations to Improve Normality or Symmetry.” Biometrika 87 (4): 954-959.

\section{AUTHOR BIOGRAPHIES}

WAEL RASHWAN, M.Sc. is a senior researcher in the 3S Group - DIT. He has a B.Sc. and M.Sc. in Operations Research from Cairo University, as well as extensive experience in industry. His research interests include Modeling and Simulation, Optimization, Game Theory and Probability Collectives. His email address iswael.rashwan@dit.ie.

HEBA HABIB, M.Sc. is a researcher in the $3 \mathrm{~S}$ Group. She has her B.Sc. and M.Sc. degrees in Operations Research and Decision Support from Cairo University. Her research interests include Decision Support, Modeling and Simulation, Optimization, Revenue Management, and Computational Intelligence. Her email address is heba.habib@dit.ie.

AMR ARISHA, $\mathrm{PhD}$ is the Director of the $3 \mathrm{~S}$ Group and the Head of Department of International Business in College of Business at the Dublin Institute of Technology (DIT), which he joined in 2005. His research interests include analysis and optimization of complex dynamic systems in manufacturing and service industries. His e-mail address is amr.arisha@dit.ie.

GARRY COURTNEY, Prof. is a consultant physician and gastroenterologist at St. Luke's Hospital, Kilkenny, and lead of the National Acute Medicine Program. His research interests include acute medicine. His e-mail address is garry.courtney@hse.ie.

SEAN KENNELLY, Dr. is a consultant physician in geriatric medicine and Director of Acute Medical Unit at Tallaght Hospital. His research interests include acute and subacute older persons hospital care, rehabilitation medicine. His e-mail address is sean.kennelly@amnch.ie. 